\title{
A study to evaluate the efficacy of combined prostaglandins and vaginal estradiol compared to prostaglandin alone in labor induction
}

\author{
Anjana B. ${ }^{1}$, Saniya Sheikh ${ }^{2 *}$ \\ ${ }^{1}$ Obstetrician, District Hospital Thoubal, Government of Manipur, Khangabok, Manipur, India \\ ${ }^{2}$ Department of Obstetrics and Gynecology, SDM Medical College and Hospital, Dharwad, Karnataka, India
}

Received: 02 January 2020

Accepted: 01 February 2020

*Correspondence:

Dr. Saniya Sheikh,

E-mail: statisticclinic2018@gmail.com

Copyright: $\odot$ the author(s), publisher and licensee Medip Academy. This is an open-access article distributed under the terms of the Creative Commons Attribution Non-Commercial License, which permits unrestricted non-commercial use, distribution, and reproduction in any medium, provided the original work is properly cited.

\begin{abstract}
Background: Induction of labor (IOL) is a process where labor is initiated artificially before its spontaneous onset for the delivery of feto placental unit by mechanical or pharmacologic methods.

Methods: The present Hospital based prospective randomized comparative study was conducted in the department of obstetrics and gynecology, KLES Dr. Prabhakar Kore Charitable Hospital, Belagavi during the period of January 2015 to December 2015.The selected women were randomized into two groups by simple randomization using an opaque sealed envelope, into either without vaginal estradiol Group A: Group PGE2 - Prostaglandin E2 gel intracervical alone group or Group B: PGE2 + E (Estradiol) - combined Prostaglandin E2 gel intracervical and $50 \mu \mathrm{g}$ of estradiol tablet intravaginal group.

Results: In the present study 65\% of the women in group PGE2 required three doses of prostaglandins compared to $23.33 \%$ in group PGE2 and estradiol for the cervix to become favorable. In this study there is significantly longer mean interval time noted for induction to cervical ripening (12.88 \pm 4.91 versus $8.92 \pm 5.07 ; \mathrm{p}<0.001)$, induction to active labor $(16.97 \pm 4.93$ versus $11.02 \pm 4.72 ; \mathrm{p}<0.001)$ and induction to delivery time $(21.97 \pm 3.83$ versus $13.14 \pm 4.98$; $\mathrm{p}<0.001$ ) in group PGE2 compared to combined PGE2 and estradiol group.

Conclusions: Thus, vaginal estradiol along with prostaglandins has the potential in cervical ripening and induce labor and in an efficacious way. There is beneficial fetal outcome when combined vaginal estradiol along with intracervical prostaglandin E2 was used.
\end{abstract}

Keywords: Estradiol, Fetal, Induction, Labour, Prostaglandin

\section{INTRODUCTION}

The aim of obstetrics is a pregnancy that results in a healthy infant and a healthy mother. Usually in majority of the women labour starts spontaneously and it results in vaginal delivery at or when the term is near. Induction of labour for cervical ripening is necessary if there are any medical or obstetric complications of pregnancy. Induction of labor is necessary when the benefits of delivery to either the mother or fetus outweigh than those who plan to continue their pregnancy. ${ }^{1}$
Induction of labour (IOL) is a process where labour is initiated artificially before its spontaneous onset for the delivery of fetoplacental unit by mechanical or pharmacologic methods. ${ }^{2}$ Though there are medical or obstetrical complications of pregnancy warranting inductions for non-medical or social reasons, induction of labour is like a rapier or a double edged sword wherein one edge of the blade is always towards the user's neck. In other words, the more interference with the normal spontaneous onset of labour the higher chances that they may land up having risks like increased caesarean 
sections, fetal distress, more instrumental deliveries, chorioamnionitis, psychological disturbances, ${ }^{3,4}$ Numerous techniques have been attempted for induction of labour to ripen the unfavourable cervix and enhance the changes necessary for labour in the lower uterine segment like intravenous infusion of oxytocin, intravaginal or intracervical administration of prostaglandins and intracervical Foley's balloon catheter insertion. ${ }^{5}$

Success of labour induction largely depends on cervical status; cervix that is unripe conveys a lower likelihood of vaginal delivery. ${ }^{6}$ When pregnant women of gestational age more than 41 weeks is induced, it is associated with a small reduction in perinatal deaths and meconium aspiration syndrome (MAS). ${ }^{7}$ Induction following PROM shows reduction in chorioamnionitis, endometritis, and neonatal ICU (NICU) admissions. ${ }^{8}$

As the pregnancy reaches term, there is increasing concentrations of estrogen in the maternal circulation, the idea that if addition of estrogen to the prostaglandins could potentiate spontaneous onset of labor has led to this study in exploring the role and efficacy of vaginal estradiol with PGE2 combination for induction of labour in unfavourable cervix and to assess the safety of the fetal outcome. Also, there is not enough literature to show the true effect of efficacy of vaginal estrogen along with prostaglandins for cervical ripening and labour induction.

Objective of this study was to evaluate the efficacy of combined prostaglandins and vaginal estradiol compared to prostaglandin alone in labour induction.

\section{METHODS}

The present Hospital based prospective randomized comparative study was conducted in the department of obstetrics and gynecology, KLES Dr. Prabhakar Kore Charitable Hospital, Belagavi during the period of January 2015 to December 2015.

The sample size was calculated by considering type I error rate $\alpha=0.05$ and type error $\beta=0.02$ and power of $10 \%$ sample size was 120 . A total of 120 pregnant women with singleton pregnancy having $\geq 36$ weeks of gestation, cephalic presentation, Bishop's score $\leq 4$, intact membrane, history of no previous Cesarean section eligible for my study for induction of labour were randomized into two groups of 60 each and studied. Singleton pregnant women $\geq 36$ weeks admitted in labour room at KLES Dr. Prabhakar Kore Charitable Hospital fulfilling inclusion criteria for induction of labour under the department of obstetrics and gynecology, KLES Dr. Prabhakar Kore Charitable Hospital, Belagavi were involved.

\section{Inclusion criteria}

- Gestational age $\geq 36$ weeks.
- Singleton pregnancy

- Cephalic presentation

- Bishop's score less than or equal to 4

- With intact membrane.

\section{Exclusion criteria}

- Pregnant women with previous LSCS or any scar on uterus

- Pregnant women with PROM/ PPROM

- Pregnant women with placenta previa.

The selected women were randomized into two groups by simple randomization using an opaque sealed envelope, into either without vaginal estradiol Group A: Group PGE2 - Prostaglandin E2 gel intracervically alone group or Group B: PGE2 + E (Estradiol) - Combined Prostaglandin E2 gel intracervically and $50 \mu \mathrm{g}$ of estradiol tablet intravaginal group. Patients were interviewed for demographic characteristics and obstetric history. Data regarding age, obstetric history and period of gestation were noted. These women were evaluated for pre-induction cervical status based on modified Bishop's Score. If pre-induction Bishop's Score $\leq 4$, they were included for the study and the findings were recorded on a predesigned and pretested preform.

\section{Group PGE2}

In this group pregnant women underwent induction of labour using intracervical prostaglandin E2 gel only, every sixth hourly for a maximum of three doses of prostaglandin till cervical ripening is achieved.

\section{Group PGE2 and estradiol (PGE2+E)}

In this group pregnant women underwent induction of labour using vaginal estradiol tablet $50 \mu \mathrm{g}$ with intracervical prostaglandin E2 gel in the first dose followed by prostaglandin E2 gel intracervically alone in the subsequent next two doses each dose after six hours apart, thereby maximum of total three doses till cervical ripening is achieved.

Induction of labor was performed by the treating obstetrician. Pre-induction Bishop's score was assessed and women who had Bishop's score $\leq 4$ were included. Non-stress test was taken for at least 20 minutes before and 30 minutes after the induction. After randomization, they were either induced using group A protocol of prostaglandin gel (dinoprostone) alone, $0.5 \mathrm{mg}$ intracervically every sixth hourly of maximum three doses till cervical ripening was achieved or, group B protocol was followed using combined prostaglandin gel $0.5 \mathrm{mg}$ intracervically and estradiol $50 \mu \mathrm{g}$ tablet vaginally inserted in the posterior fornix in the first dose followed by prostaglandin gel $0.5 \mathrm{mg}$ intracervically in the subsequent next two doses every sixth hourly for total of maximum three doses till cervical ripening was 
achieved. Women were advised to be at bed for at least half an hour after induction.

\section{Statistical analysis}

Data obtained was coded and entered into the Microsoft Excel Spreadsheet. The data was analyzed using statistical software SPSS 20. The categorical data was expressed in terms of frequencies and percentages while continuous data was expressed as mean \pm standard deviation (SD). The two groups were compared using either chi-square test or Fishers exact test for categorical data and independent sample's' test was used to compare the means of different parameters. A ' $p$ ' value of less than or equal to 0.05 at 95 Confidence intervals was considered as statistically significant.

\section{RESULTS}

These women were randomized into two groups of 60 each, as Group PGE2 (induction with intracervical prostaglandin E2 gel only every 6th hourly of maximum three doses) and Group PGE2 and vaginal estradiol (induction with $50 \mu \mathrm{g}$ estradiol tablet vaginally with intracervical prostaglandin E2 gel in the first dose followed by prostaglandin E2 gel intracervically alone in further next two doses, sixth hourly interval of maximum three doses in total till cervical ripening is achieved).

Table 1: Distribution of social and pregnancy profile in both the groups.

\begin{tabular}{|c|c|c|c|c|c|c|}
\hline & & \multicolumn{2}{|c|}{ Group PGE2 $(n=60)$} & \multicolumn{2}{|c|}{ Group PGE2+E $(n=60)$} & \multirow{2}{*}{ p value } \\
\hline & & Number & $\%$ & Number & $\%$ & \\
\hline \multirow{4}{*}{ Age group } & 20 or less & 10 & 16.67 & 13 & 21.67 & \multirow{4}{*}{0.438} \\
\hline & 21 to 25 & 35 & 58.33 & 27 & 45.00 & \\
\hline & 26 to 30 & 13 & 21.67 & 15 & 25.00 & \\
\hline & 30 or more & 2 & 3.33 & 5 & 8.33 & \\
\hline \multirow{2}{*}{ Antenatal care } & Unregistered & 26 & 43.33 & 37 & 61.67 & \multirow{2}{*}{0.577} \\
\hline & Registered & 34 & 56.67 & 23 & 38.33 & \\
\hline \multirow{6}{*}{ Gravida } & Primi & 41 & 68.33 & 32 & 53.33 & \multirow{6}{*}{0.210} \\
\hline & 2 & 12 & 20.00 & 11 & 18.33 & \\
\hline & 3 & 5 & 8.33 & 10 & 16.67 & \\
\hline & 4 & 1 & 1.67 & 5 & 8.33 & \\
\hline & 5 & 1 & 1.67 & 1 & 1.67 & \\
\hline & 6 & 0 & 0.00 & 1 & 1.67 & \\
\hline \multirow{2}{*}{ Parity } & Primigravida & 41 & 68.33 & 32 & 53.33 & \multirow{2}{*}{0.092} \\
\hline & Multigravida & 19 & 31.66 & 28 & 46.66 & \\
\hline \multirow{6}{*}{ Gestational weeks } & $36+1$ to $36+6$ & 3 & 5.00 & 3 & 5.00 & \multirow{6}{*}{0.659} \\
\hline & $37+1$ to $37+6$ & 12 & 20.00 & 6 & 10.00 & \\
\hline & $38+1$ to $38+6$ & 10 & 16.67 & 9 & 15.00 & \\
\hline & $39+1$ to $39+6$ & 9 & 15.00 & 13 & 21.67 & \\
\hline & $40+1$ to $40+6$ & 17 & 28.33 & 19 & 31.67 & \\
\hline & $41+1$ to $41+6$ & 9 & 13.33 & 10 & 11.67 & \\
\hline
\end{tabular}

In the present study most of the women were aged between 21 to 25 years in group in PGE2 $(58.33 \%)$ as well as in group PGE2 and estradiol (45\%). However, the age distribution in group PGE2 and group PGE2 and estradiol was comparable $(\mathrm{p}=0.438)$. The mean age in group PGE2 was 23.67 \pm 3.05 years compared to $24.53 \pm 3.98$ years in group PGE2 and estradiol. In the present study $56.67 \%$ of the women in group PGE2 and $38.33 \%$ in group PGE2 and estradiol was registered case for antenatal care. However, both the groups were comparable in terms of antenatal care $(p=0.577)$. In the present study most of the women in group PGE2 $(68.33 \%)$ as well as PGE2 and estradiol (63.33\%) were primigravida $(\mathrm{p}=0.210)$ and hence comparable. In this study $28.33 \%$ of the women from group PGE2 and
$31.67 \%$ from group PGE2 and estradiol had gestational age between $40 \pm 1$ to $40 \pm 6$ weeks $(p=0.659)$ and hence were comparable. In this study the mean period of gestation in group PGE2 was 39.24 \pm 1.47 weeks compared to $39.54 \pm 1.51$ weeks in group PGE2 and estradiol but the difference observed was statistically not significant $(\mathrm{p}=0.252)$ (Table 1$)$.

In the present study the commonest indication for induction of labor was postdatism in group PGE2 $(21.67 \%)$ as well as group PGE2 and estradiol (26.67\%).

In the present study pre induction Bishop's Score of 3 and 4 were noted maximum in both the groups i.e., $28.33 \%$ and $20 \%$ respectively in PGE2 group and in 
PGE2 and estradiol group as $26.67 \%$ and $28.33 \%$. Pre induction Bishop's Score of 2 were similar in both study groups to be $20 \%$. Pre induction Bishop's Score of 1 was $16.67 \%$ in PGE2 group and $13.33 \%$ in combined group. Pre induction Bishop's Score of zero were least in both study groups as $15 \%$ and $11.67 \%$ in combined study group. However, the distribution of women based on pre induction Bishop's Scores was almost equal and was not significant $(p=0.850)$. In this study the mean pre induction Bishop's score in group PGE2 was 2.19 \pm 1.37 compared to $2.45 \pm 1.35$ in group PGE2 and estradiol (Table 2).

In the present study $65 \%$ of the women in group PGE2 required three doses of prostaglandins compared to $23.33 \%$ in group PGE2 and estradiol for the cervix to become favourable. In this study the mean number of doses administered were significantly higher in PGE2 group $(2.62 \pm 0.59)$ compared to that of combined PGE2 and estradiol group $(1.86 \pm 0.76)$ which was statically significant. In the present study the significantly higher number of women had successful cervical ripening in group PGE2 and estradiol $(83.33 \%)$ compared to group
PGE2 (61.67\%). 38.33\% (30/120) of women in both the groups had failed cervical ripening $(\mathrm{p}=0.001)$. 7 failed to achieve active labour in PGE2 group and 2 failed to achieve establishment of active labour in combined group. In the present study higher number of vaginal deliveries were noted in PGE2 and vaginal group significantly compared to PGE2 alone group (80\% versus $51.67 \%)$ and hence was statistically significant $(\mathrm{p}=$ 0.001 ) (Table 3).

Table 2: Pre-induction Bishop's score.

\begin{tabular}{|lllll|}
\hline \multirow{2}{*}{ Score } & \multicolumn{2}{l}{$\begin{array}{l}\text { Group PGE2 } \\
(\mathbf{n = 6 0 )}\end{array}$} & \multicolumn{2}{l|}{$\begin{array}{l}\text { Group PGE2 +E } \\
(\mathbf{n = 6 0})\end{array}$} \\
\cline { 2 - 5 } & Number & $\%$ & Number & \% \\
\hline 0 & 9 & 15.00 & 7 & 11.67 \\
\hline 1 & 10 & 16.67 & 8 & 13.33 \\
\hline 2 & 12 & 20.00 & 12 & 20.00 \\
\hline 3 & 17 & 28.33 & 16 & 26.67 \\
\hline 4 & 12 & 20.00 & 17 & 28.33 \\
\hline Total & $\mathbf{6 0}$ & $\mathbf{1 0 0 . 0 0}$ & $\mathbf{6 0}$ & $\mathbf{1 0 0 . 0 0}$ \\
\hline
\end{tabular}

Chi square $=1.365 \mathrm{p}=0.850$.

Table 3: Comparison of labor parameters among study subjects in both the groups.

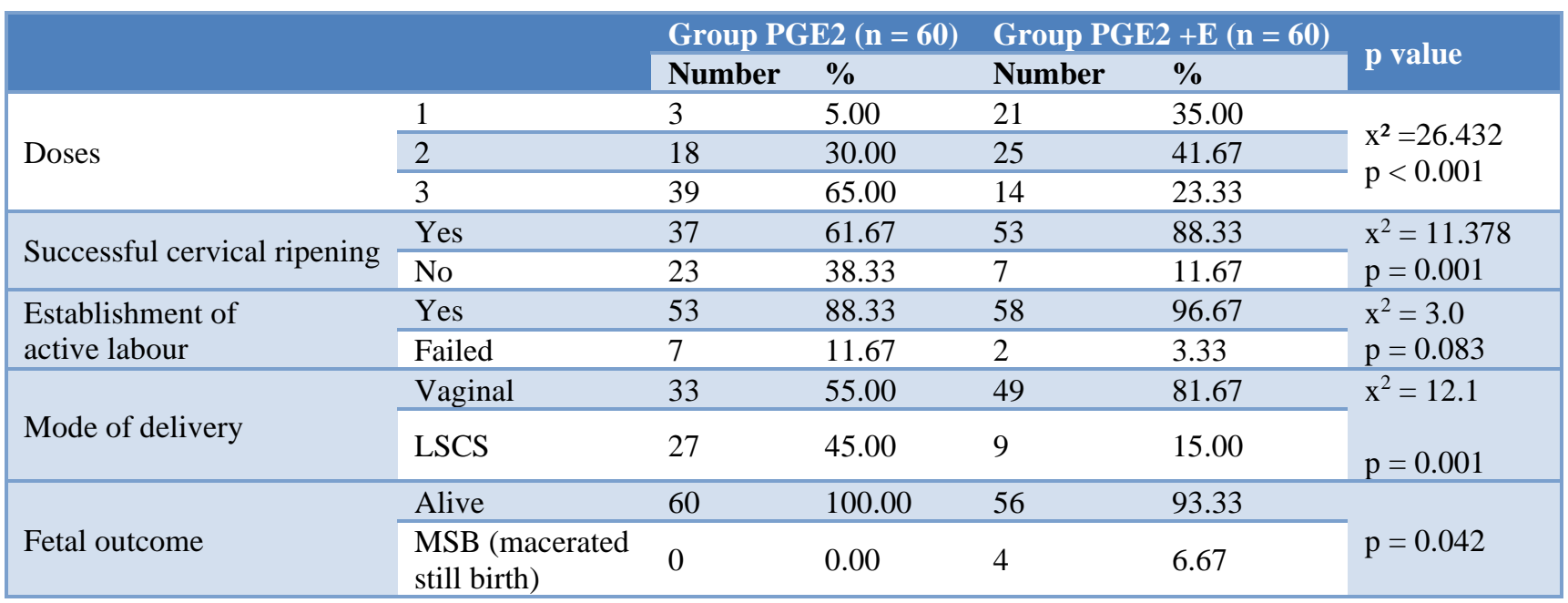

Table 4: Mean time interval for induction of labour.

\begin{tabular}{|c|c|c|c|c|c|}
\hline \multirow{2}{*}{ Variables } & \multicolumn{2}{|c|}{ Group PGE2 $(n=60)$} & \multicolumn{2}{|c|}{ Group PGE2+E $(n=60)$} & \multirow{2}{*}{ p value } \\
\hline & Mean & SD & Mean & SD & \\
\hline Induction to cervical ripening interval (hours) & 12.88 & 4.91 & 8.92 & 5.07 & $<0.001$ \\
\hline Induction to active labour interval (hours) & 16.97 & 4.93 & 11.02 & 4.72 & $<0.001$ \\
\hline Time for from induction to delivery (hours) & 21.97 & 3.83 & 13.14 & 4.98 & $<0.001$ \\
\hline
\end{tabular}

In this study there is significantly longer mean interval time noted for induction to cervical ripening $(12.88 \pm 4.91$ versus $8.92 \pm 5.07 ; \mathrm{p}<0.001)$, induction to active labour $(16.97 \pm 4.93$ versus $11.02 \pm 4.72 ; \mathrm{p}<0.001)$ and induction to delivery time $(21.97 \pm 3.83$ versus $13.14 \pm 4.98$; $p$ $<0.001)$ in group PGE2 compared to combined PGE2 and estradiol group. Hence, they were statistically significant in each time interval.

\section{DISCUSSION}

The incidence of labour induction has increased over the last decade. Labour induction may be indicated by 
medical or obstetrical complications of pregnancy or may be requested or chosen for non-medical or social reasons. Around $20 \%$ of all deliveries are preceded by labor induction. In the last 50-60 years, prolonged pregnancy and maternal hypertensive disorders were the major indications.

In a prospective double-blind study by Dasgupta $E$ and Singh $\mathrm{G}$, the mean maternal age was $21.67 \pm 1.15$ years in women who underwent induction of labour with misoprostol compared to women who underwent induction of labour with misoprostol + estradiol had mean age of $22.33 \pm 2.58$ years $(p=0.121) .{ }^{9}$ The mean maternal age observed in the present study was slightly higher in both the groups compared to the study by Dasgupta E and Singh G but was comparable in both the groups. $^{9}$

The mean number of doses of prostaglandins administered were significantly higher in PGE2 only group compared to PGE2 and estradiol combined group (2.62 \pm 0.59 versus $1.86 \pm 0.75 ; \mathrm{p}<0.001)$. These findings suggest that, induction of labour with intracervical prostaglandin E2 gel requires significantly higher number of doses compared to combined intravaginal estradiol tablet and prostaglandin E2 gel. On an average, 4-5 doses of misoprostol were required in a study by Dasgupta $\mathrm{E}$ and Singh $\mathrm{G}$, for cervical ripening or initiation of active labor which is similar to other studies also, however dose required in combined group was significantly less $(\mathrm{p}=$ 0.017). ${ }^{9}$ In study by Dasgupta $E$ and Singh $G$, in misoprostol only group, duration of interval between induction initiation to cervical ripening, induction initiation to active labor and initiation and induction initiation to delivery were $12.67 \pm 2.21,15.33 \pm 3.76$ and $18.25 \pm 6.13$ hours, respectively. 9,10

According to Raksha M and Rao A study, when vaginal misoprostol was combined with vaginal estradiol the mean time interval from induction to cervical ripening had shown to be 7.62 hours which is in slightly similar to our study, it took 8.92 hours for combined prostaglandins and vaginal estradiol group for cervix to ripen after initial induction. ${ }^{10}$ In Crane JMC, Butler B a systemic review study, there was a higher rate of vaginal deliveries with misoprostol compared to PGE2 $(\mathrm{RR}=1.22,95 \% \mathrm{CI}=$ 0.96-1.55) and lower rate of oxytocin administered in misoprostol group when compared to PGE2 group (46.4\% versus $62.4 \%$ ). Various studies have found induction delivery interval with vaginal misoprostol in Dasgupta E and Singh G to be 16-20 hours, which is in agreement with our study $(16.97 \pm 4.93) .{ }^{9}$ In Dasgupta E and Singh G study and Raksha M and Rao A study, combined misoprostol and vaginal estradiol the induction to delivery duration interval were reduced to 12.07 hours while in present study it took 13.14 hours in combined group as compared to prostaglandin alone group which was much more i.e., 21.97 hours. ${ }^{9,10}$
Some studies have shown effect on cesarean section rate with misoprostol as in Raksha M and Rao A study, they had 29 caesareans with misoprostol group and 10 with misoprostol and estradiol group. ${ }^{10}$ In the present study significantly higher number of vaginal deliveries were noted in group PGE2 and estradiol compared to group PGE2. However, no such difference was found in Dasgupta E and Singh G study. ${ }^{9}$

\section{CONCLUSION}

Based on the findings of this study it may be concluded that, induction of labor with combined vaginal estradiol along with intracervical prostaglandin E2 gel requires significantly lower number of doses of prostaglandin E2 gel for cervical ripening, yields higher success rate of cervical ripening with lesser duration interval for establishment of active labor and induction to delivery time and is more effective that of intracervical prostaglandin E2 gel alone. Thus, vaginal estradiol along with prostaglandins has the potential in cervical ripening and induce labor and in an efficacious way. There is beneficial fetal outcome when combined vaginal estradiol along with intracervical prostaglandin E2 was used.

\section{Funding: No funding sources Conflict of interest: None declared \\ Ethical approval: The study was approved by the Institutional Ethics Committee}

\section{REFERENCES}

1. Galal M, Symonds I, Murray H, Petraglia F, Smith R. Postterm pregnancy. Facts Views Vis Obgyn. 2012;4(3):175-87.

2. Deshmukh VL, Yelikar KA, Waso V. Comparative Study of efficacy and safety of oral versus vaginal misoprostol for induction or labour. J Obstet Gynaecol India. 2013;63(5):321-4.

3. Abdel Hak AM, Hanafy A. Pre-induction cervical ripening with Prostaglandin E2 in Preterm Pregn. Med J Cairo Univ. 2010;78(1):307-11.

4. Mylonas I, Friese K. Indications for and risks of elective caesarean section. Dtsch Arztebl Int. 2015;112(29-30):489-95.

5. Mozurkewich EL, Chilimigras JL, Berman DR, Perni UC, Romero VC, King VJ, et al. Methods of induction of labor: a systematic review. BMC Preg Childbirth. 2011;11:84.

6. Shah K, Doshi H. Premature rupture of membrane at term: early induction versus expectant. Management 2012;62(2):172.

7. Bernstein P. Prostaglandin E2 gel for cervical ripening and labour induction: A multi-center placebo-controlled trial. CMAJ. 1991;145(10):124954.

8. Ulmsten U, Wingerup L, Ekman G. Local application of prostaglandin E2 for cervical ripening or induction of term labor. Clin Obstet Gynecol. 1983;26(1):95-105. 
9. Dasgupta E, Singh G. Vaginal misoprostol vs vaginal misoprostol with estradiol for labor induction: a prospective double-blind study. 2012; 62(1): 47-51.

10. Leduc D, Bringer A, Lee L. Induction of labour. SOGC Clin Pract Guideline Induct Labor. 2013;296:840-58
Cite this article as: Anjana B, Sheikh S. A study to evaluate the efficacy of combined prostaglandins and vaginal estradiol compared to prostaglandin alone in labor induction. Int J Reprod Contracept Obstet Gynecol 2020;9:1153-8. 